\title{
Results from the first year of the INTEGRAL Spiral Arms Monitoring Program
}

\author{
Arash Bodaghee*, on behalf of the ISA Collaboration \\ UC Berkeley - Space Sciences Laboratory \\ E-mail: bodagheedssl.berkeley.edu
}

The INTEGRAL Spiral Arms (ISA) Key Program consists of high-cadence monitoring of the inner Galactic spiral arms (1.2 Ms in AO9; 0.6 Ms in AO10) combined with follow-up observations of new IGRs with Swift for position refinement and an initial spectrum (5-10 ToOs of $2 \mathrm{ks}$ ) and with XMM-Newton for deeper timing and spectral analysis (3-5 ToOs of $10 \mathrm{ks}$ ). The regions of interest are the Inner Perseus/Norma Arm tangents on one side of the Galactic Center, and the Scutum/Sagittarius Arms on the other. Each arm region is observed for $12.6 \mathrm{ks}$ per spacecraft revolution during visibility periods. While these regions are already exposed thanks to the Galactic Plane Scans (GPS), many transients are being missed because of month-long gaps between scans of any particular region. These fields feature a high density of obscured high-mass X-ray binaries (HMXBs), including Supergiant Fast X-ray Transients (SFXTs), as well as other hard X-ray emitting sources (e.g. microquasars, low-mass X-ray binaries, and magnetars) that INTEGRAL is well-suited to finding thanks to its large field of view and angular resolution at high energies even in crowded regions of the sky. Our primary objectives are an increase in the discovery rate of new X-ray transients and better monitoring of known sources. The data are public but we provide mosaic images and source light curves in 4 energy bands (from ISGRI and JEM-X) to the community at http://ssl.berkeley.edu/ bodaghee/isa.

An INTEGRAL view of the high-energy sky (the first 10 years) - 9th INTEGRAL Workshop and celebration of the 10th anniversary of the launch

15-19 October 2012

Bibliotheque Nationale de France, Paris, France

\footnotetext{
${ }^{*}$ Speaker.
} 


\section{Introduction}

The inner spiral arms feature the highest formation rates of massive OB stars in the Milky Way. Some of these stars evolve to become high-mass X-ray binaries (HMXBs) that populate the dust-obscured regions of the Galactic Plane. Because HMXBs feature a compact object that accretes from a massive donor star $\left(\gtrsim 10 M_{\odot}\right)$, they represent laboratories for studying accretion physics under extreme gravitational and electromagnetic fields, and they serve as tracers of the recent star-formation history of the Milky Way.

Photoelectric absorption along the Galactic Plane, especially towards the spiral arm tangents where HMXBs tend to cluster, blocks X-rays below $\sim 5 \mathrm{keV}$ which means that HMXBs can be difficult to detect in soft X-ray surveys (e.g., Rosat, Swift-XRT). Operating above $\sim 20 \mathrm{keV}$ allows the soft gamma-ray imager INTEGRAL-ISGRI to peer through this obscuring material. Thanks to its wide field of view (FoV) and spatial resolution in the hard X-rays, INTEGRAL has been instrumental in discovering HMXBs - more than doubling the number of known systems in the Galaxy. Many of them are transient emitters (supergiant fast X-ray transients or SFXTs) that undergo brief and unpredictable flaring episodes, so follow-up observations must be performed soon (i.e., within several hours or days) after their discovery. Otherwise, the high-energy emission drops to the quiescent levels that are often too faint to be detected by even the most sensitive X-ray telescopes.

Hence, the best way to discover these kinds of sources and to characterize their behavior is through a campaign dedicated to the hard X-ray monitoring of their likely host regions in tandem with rapid follow-up observations from soft X-ray telescopes. The INTEGRAL Spiral Arms (ISA) program represents such a campaign. We will search for new transient HMXBs where they are likely to be found, i.e., the inner spiral arms of the Milky Way, and new hard X-ray sources will be observed in the soft X-rays thanks to approved Target of Opportunity (ToO) observations with Swift and XMM-Newton. While these regions are already exposed thanks to the Galactic Plane Scans (GPS; PI: Bazzano), many transients are being missed because of month-long gaps between scans of any particular region, and because the GPS devotes as much time to the Galactic anticenter as it does to source-rich regions of the inner disk. In essence, the ISA program increases the observing frequency of scientifically-valuable regions of the plane during viewable periods compared with the GPS (which remains useful for the rest of the plane).

The objectives of the ISA program are an improved sampling of the transient population in the spiral arms and a more complete picture of the number, distribution, and behavior of accreting compact objects in our Galaxy.

\section{The ISA program}

Our observing program called "INTEGRAL Monitoring of the Inner Spiral Arms" (PI: Bodaghee) began in INTEGRAL cycle 9 (2012), and has been approved for continuation into cycle 10 (2013). This program consists of $1.2 \mathrm{Ms}$ worth of regular scans by INTEGRAL of the inner spiral arm tangents of the Galaxy. The Region of Interest (RoI) is divided into two large fields situated on opposite sides of the Galactic Center (GC) from our point of view: the tangent to the Inner Perseus and Norma arms on the right side of the GC at Galactic coordinates $(l ; b)=\left(337.5^{\circ} ; 0^{\circ}\right)$; and the tangent to the Scutum and Sagittarius arms on the left side of the GC at $(l ; b)=\left(22.5^{\circ} ; 0^{\circ}\right)$. The centers 


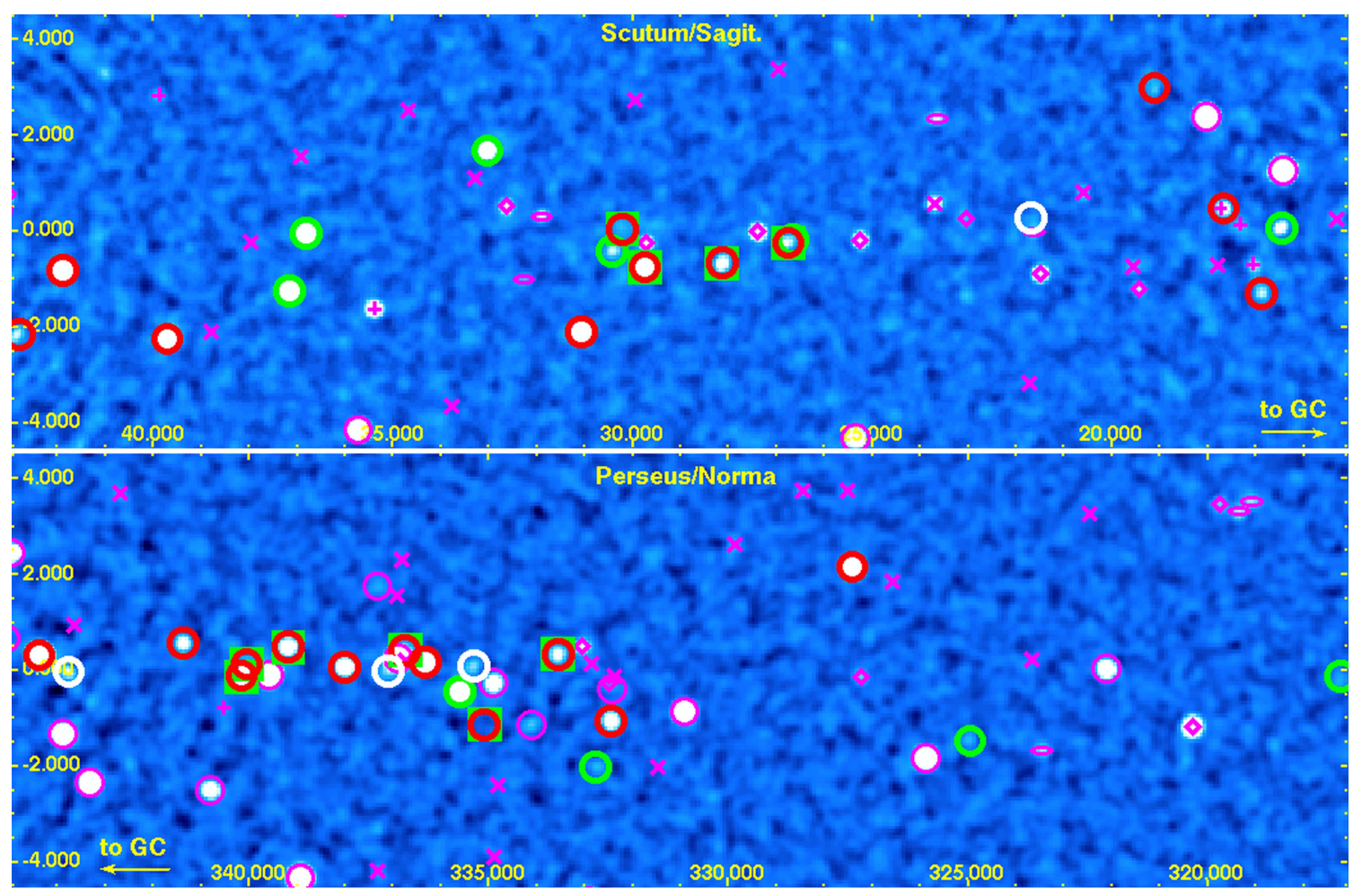

Figure 1: INTEGRAL-ISGRI significance maps (3 Ms; $18-40 \mathrm{keV}$ ) of a section of the spiral arm tangents targeted in the ISA program along with the locations of all sources detected by ISGRI to date. The upper panel presents the Scutum/Sagittarius Arms, while the lower panel displays the Inner Perseus/Norma Arms. The arrows indicate the direction to the Galactic Center. Circles represent low and high-mass X-ray binaries: red for SGXBs (the SFXTs are boxed); green for BEXBs; white for unclassified HMXBs; and magenta for LMXBs. Unclassified X-ray binaries are shown as magenta plus symbols. Other Galactic objects (cataclysmic variables, magnetars, supernova remnants, etc.) are labelled with magenta diamonds. Extragalactic objects are denoted by magenta ellipses. Magenta crosses symbolize sources awaiting classification.

of these fields were chosen to maximize the number of ISGRI-detected HMXBs in a $15^{\circ}$ bin of longitude which is roughly the width of the ISGRI fully-coded FoV.

The density of hard X-ray sources in the inner spiral arms is illustrated in Fig. 1. There are 36 HMXBs in this image which represents less sky area than the ISGRI partially-coded FoV. The targeted regions $\left(50^{\circ}\right.$-wide combined, i.e., wider than that shown in Fig. 1) contain at least 3 times as many hard X-ray detected HMXBs per longitude degree (0.7 HMXBs/deg) compared with the average for the rest of the Galactic Plane (0.2 HMXBs/deg). The SFXT population is even more confined to the arms: of $\sim 20$ candidate SFXTs in the Galaxy, 14 are located within the inner spiral arms. This amounts to $0.3 \mathrm{SFXTs} / \mathrm{deg}$ in the arms compared with $0.02 \mathrm{SFXTs} / \mathrm{deg}$ for the rest of the plane. The survey regions contain hundreds of other sources, around half of which were discovered by INTEGRAL.

For each spacecraft revolution ( 3 days), INTEGRAL observes one (or both) of these fields as long as they are visible: the visibility period lasts 4-6 non-contiguous months per year. Each observation lasts $12.6 \mathrm{ks}$ consisting of seven pointings of $1.8 \mathrm{ks}$ arranged in a hexagonal pattern 

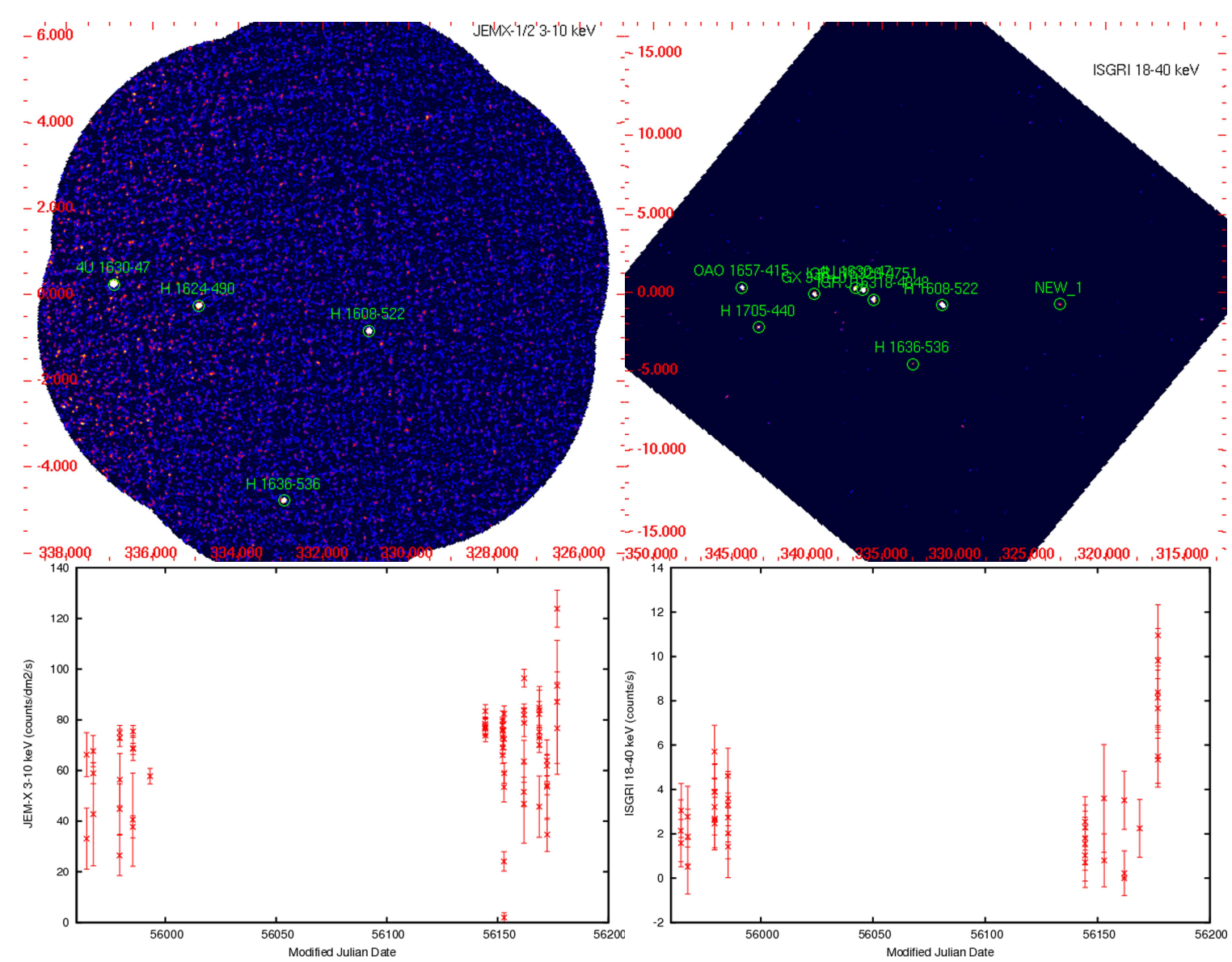

Figure 2: Examples of reduced data products from the ISA progrom deomnstrating how X-ray sources are monitored in several energy bands and over the long term. The top row presents JEM-X $(3-10 \mathrm{keV})$ and ISGRI (18-40 keV) significance maps in Galactic coordinates from INTEGRAL revolution 1143 (MJD 55979.377). The bottom row displays the X-ray light curves (in the same energy bands as above) for one of the detected sources, $4 \mathrm{U} 1630-47$, using all ISA observations to date. These figures, and similar images and light curves from other sources, are available online (http://ssl.berkeley.edu/ bodaghee/isa).

with one pointing at each corner of the hexagon and one at the center. The center of the hexagonal pattern is offset by $1^{\circ}$ after each observation in order to overlap JEM-X and ISGRI pointings and to reduce imaging systematics. The limiting sensitivity in the fully-coded FoV is $\sim 10 \mathrm{mCrab}$ in the JEM-X and ISGRI bands after a single hexagonal observation, and $\sim 5 \mathrm{mCrab}$ at the end of the entire program. We elected to make all data public, but we also provide mosaic images and light curves of detected sources in 4 energy bands (3-10, 10-25, 18-40, and 40-100 keV) soon after each observation is completed. Figure 2 presents examples of reduced data products from JEM-X and ISGRI.

Among the benefits of the ISA program is that it provides a higher observing cadence of known variable sources in the spiral arms, permitting an improved study of their long-term timing and spectral evolution. Variable sources include HMXBs, but also: low-mass X-ray binaries (LMXBs); microquasars ( $\mu \mathrm{QSOs}$ ); cataclysmic variables (CVs); anomalous X-ray pulsars (AXPs), magnetars, and soft-gamma repeaters (SGRs); and active galactic nuclei (AGN) viewed through the 


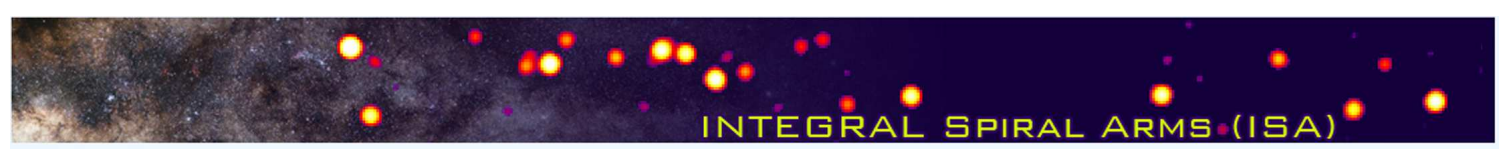

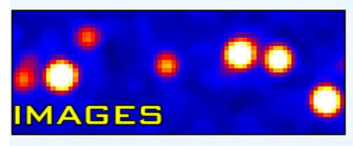
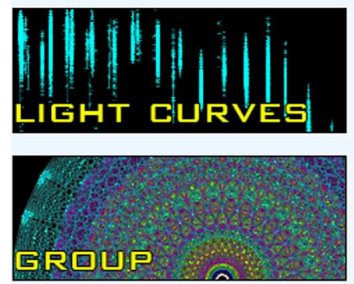

INTEGRAL Spiral Arms (ISA)

The INTEGRAL Spiral Arms (ISA) Monitoring Program consists of regular observations of the Scutum and Norma Arm regions (Galactic Longitude: +-25 degrees).

Here, you will find images of the fields and a list of sources detected during the ISA program.

Abstract: A Key Programme is proposed for high-cadence monitoring of the inner spiral arms of the Galaxy with INTEGRAL paired with ToO observations of new transients with XMM-Newton and Swift. While these regions are already exposed thanks to the Galactic Plane Scans (GPS), many transients are being missed because of month-long gaps between scans of any particular region. The proposed program ( $12.3 \mathrm{ks}$ per arm region, when visible, for a total of $1.2 \mathrm{Ms}$ during AO9) will complement the successful Galactic bulge (GB) monitoring program by extending the monitored region of the Galaxy to the Inner Perseus/Norma Arm tangents on one side of the GB $(I, b)=(337.5,0)$, and the Scutum/Sagittarius Arms on the other Inner Pe $(1, b)=(22.5,0)$. These fids are ne with obscured high-mass $X$-ray binaries (HMXBs), Supergiant Fast X-ray Transients (SF that INIEGRAL is well-sult to finding thanks to its iarge, migh (issemination of results which will enable prompt follow-up of interesting events.

Schedule \& Results

Current INTEGRAL revolution

Current INTEGRAL observations

Planned INTEGRAL observations

\begin{tabular}{|l|l||l||l|}
\hline Rev. & Dates & Target & Comments (X=executed; $P=$ planned) \\
\hline \hline 1138 & 2012-02-07 10:05:24 to 2012-02-07 14:11:29 & Perseus/Norma & $X$ \\
\hline
\end{tabular}

Figure 3: Web page of the ISA program located at http://sprg.ssl.berkeley.edu/ bodaghee/isa. The main panel presents the welcome page which lists the main results. From here, one can find images of each observation from ISGRI (18-40 and 40-100 keV) and from JEM-X (3-10 and 10-25 keV), as well as light curves in those bands for all detected sources.

Galactic Plane. Known sources in the direction of the spiral arms are observed more often (in both total exposure and in the frequency of the observations) than they are currently with the INTEGRAL GPS. Having additional data points between what are otherwise long gaps in the source light curve allows us to track their luminosity variations (which are related to changes in the accretion rate). These extra observations enlarge the baseline making it easier to locate periodic signals such as pulsation and orbital periods which tell us about the nature of the compact object and the geometry of the system, respectively. We will add to the INTEGRAL monitoring of the Galactic Bulge [1] by extending the observations to higher longitudes which will be useful when comparing the transient population of the bulge with that of the disk. The timing and spectral data from our INTEGRAL observations complement those of existing hard X-ray monitors MAXI and Swift.

\section{Preliminary results}

Around $40 \%$ of ISA observations ( $470 \mathrm{ks}$ of $1.2 \mathrm{Ms}$ ) for AO9 have been performed thus far and reduced data products are available at http://sprg.ssl.berkeley.edu/ bodaghee/isa (Fig. 3). Most of our observations were interrupted or delayed due to time-sensitive observations of SN 2011fe and due to a large Solar flare in 2012 March. As a result, an estimated $730 \mathrm{ks}$ of our program in AO9 will be carried over into AO10. Furthermore, ISA has been approved for continuation into AO10. This means a total exposure time of $1.3 \mathrm{Ms}$ is dedicated to ISA observations during 2013. 
While we have not yet discovered a new transient, our observing strategy has proven successful in achieving our other objectives. On top of the wealth of light curve and spectral data that we have accumulated for the $\sim 600 \mathrm{X}$-ray sources in the RoI, we were able to confirm high-energy emission from the microquasar candidates 4U 1630-47, IGR J17091-3624, and Swift J1910.2-0546 during their recent hard states $[2,3,4]$. The discovery of the hard X-ray transient IGR J18179-1621 [5] preceded our scheduled ISA observations by a few hours. However, the detection of a cyclotron feature [6] was made possible thanks to ISA monitoring observations of the Scutum/Sagittarius Arms in subsequent INTEGRAL revolutions.

\section{References}

[1] Kuulkers, E., Shaw, S. E., Paizis, A., et al. 2007, A\&A, 466, 595

[2] Bodaghee, A., Popp, A., Grinberg, V., et al. 2012, ATel, 3945, 1

[3] Bodaghee, A., Bozzo, E., Tomsick, J. A., et al. 2012, ATel, 4328, 1

[4] Bodaghee, A., Kuulkers, E., Tomsick, J. A., et al. 2012, ATel, 4360, 1

[5] Tuerler, M., Chenevez, J., Bozzo, E., et al. 2012, ATel, 3947, 1

[6] Bozzo, E., Ferrigno, C., Türler, M., Manousakis, A., \& Falanga, M. 2012, A\&A, 545, A83 